\title{
Pilgrims' Health Screening Profile from a FELDA Settlement in Pekan, Pahang
}

Nor Azam Kamaruzaman

Department of Family Medicine, Kulliyah of Medicine, International Islamic University Malaysia Kuantan, Pahang, Malaysia

Introduction: Good physical health or istitha'ah is one of the shariah requisite to perform hajj pilgrimage. Hence, passing the health screening is mandatory for the pilgrim to-be in Malaysia. The aim of this study is to describe the pilgrims' health screening profile from a FELDA settlement in Pekan, Pahang. Materials and method: All 26 candidates from Chini's FELDA cluster who were selected for hajj pilgrimage in 2017 underwent health screening at a nearby health clinic. Data from this health screening were analysed descriptively using SPSS software version 23.0. Results: Candidates consists of 11 men and 15 women aged between 48 to 86 years with the mean age of 61 . Result showed that both sexes have comparable mean age and body mass index. Most of the candidates (88.5\%) have at least 1 chronic medical problem (excluding overweight) while $54 \%$ have at least 2 or more medical problems. Women have higher prevalence of non-communicable disease (NCD) conditions namely obesity, hypertension, and diabetes $(40 \%, 60 \%$, and $40 \%)$ compared to men $(27 \%, 45 \%$ and $36 \%$ respectively). This figure is higher than 2015 national figure for age specific: 50 years group (obesity $36.6 \%$, hypertension $49.3 \%$, diabetes $27 \%$ ). In addition, $20 \%$ of the women also have anaemia while none in men. Conclusion: Majority of the candidates in this group are elderly and have at least two chronic medical problems. Women have higher prevalence of NCD and anaemia than men. Thus, based on the findings, early and focused pilgrims' health screening especially among elderly female is essential and appropriate action should be taken in order to reduce risk for morbidity and mortality during hajj pilgrimage. 